\title{
Improving the color switch contrast in PEDOT:PSS-based electrochromic displays
}

\author{
Jun Kawahara, Peter Andersson Ersman, Isak Engquist and Magnus Berggren
}

\section{Linköping University Post Print}

N.B.: When citing this work, cite the original article.

Original Publication:

Jun Kawahara, Peter Andersson Ersman, Isak Engquist and Magnus Berggren, Improving the color switch contrast in PEDOT:PSS-based electrochromic displays, 2012, Organic electronics, (13), 3, 469-474.

http://dx.doi.org/10.1016/j.orgel.2011.12.007

Copyright: Elsevier

http://www.elsevier.com/

Postprint available at: Linköping University Electronic Press

http://urn.kb.se/resolve?urn=urn:nbn:se:liu:diva-74723 
Improving the color switch contrast in PEDOT:PSS-based electrochromic displays

Jun Kawahara ${ }^{\mathrm{a}, \mathrm{b}, \mathrm{c}}$, Peter Andersson Ersman ${ }^{\mathrm{a}}$, Isak Engquist ${ }^{\mathrm{c}}$ and Magnus Berggren ${ }^{*, c}$

${ }^{a}$ Dept. of Printed Electronics, Acreo AB, Box 787, SE-60117, Norrköping, Sweden

${ }^{\mathrm{b}}$ R\&D Strategy Dept., Lintec Corporation, Warabi, Saitama, Japan

${ }^{c}$ Organic Electronics, Dept. of Science and Technology, Linköping University, Norrköping, Sweden

* Corresponding author: Tel. +46 11 363637, e-mail address: magnus.berggren@liu.se

Abstract:

Poly(3,4-ethylenedioxythiophene) chemically doped with poly(styrene sulfonic acid) (PEDOT:PSS) is a material system commonly used as a conductive and transparent coating in several important electronic applications. The material is also electrochemically active and exhibits electrochromic (EC) properties making it suitable as the active element in EC display applications. In this work uniformly coated PEDOT:PSS layers were used both as the pixel electrode and as the counter electrode in EC display components. The pixel and counter electrodes were separated by a whitish opaque and water-based polyelectrolyte and the thicknesses of the two EC layers were varied independently in order to optimize the color contrast of the display element. A color contrast $\left(\Delta \mathrm{E}^{*}, \mathrm{CIE} \mathrm{L}^{*} \mathrm{a}^{*} \mathrm{~b}^{*}\right.$ color space) exceeding 40 was obtained with maintained relatively short switching time at an operational voltage less than $2 \mathrm{~V}$.

Keywords:

PEDOT:PSS, electrochromic displays, color contrast, printed electronics, flexible displays 


\section{Introduction}

All since the first conductive polymer, polyacetylene [1], was discovered in the late 1970s, various electroactive polymer materials have been synthesized and studied. Polythiophene and its different derivatives is a popular class of materials and have been widely explored in electronic applications, in part because of their good solubility, processability and stability [2]. The electronic conductivity of polythiophenes can typically be elevated upon electrochemical or chemical doping with counter ions. For example, the electronic conductivity of poly(3-dodecylthiophene) can approach $1000 \mathrm{~S} / \mathrm{cm}$ by doping with iodine [3]. Further, doping using polyanions, such as poly(styrene sulfonic acid) (PSS), has been explored to achieve stable, highly conductive and water-processable polymer systems $[4,5,6,7]$, and today such polyelectrolyte-doped conjugated polymer systems are widely used and explored in a wide range of different electronic applications [ 8 ]. During the last decade such material systems have commonly incorporated poly(3,4-ethylenedioxythiophene) (PEDOT) as the electronically conducting phase.

Thanks to its electrochromic feature a variety of metal oxides, monomers and polymers, e.g. $\mathrm{WO}_{\mathrm{x}}$, viologen and PEDOT:PSS, have been explored as the dynamic colorant in several applications, such as in smart windows and displays $[8,9,10,11]$. The electrochromic color-switching feature of PEDOT:PSS accompanies the following electrochemical reaction:

$$
\mathrm{PEDOT}^{+} \mathrm{PSS}^{-}+\mathrm{M}^{+}+\mathrm{e}^{-} \leftrightarrow \quad \mathrm{PEDOT}^{0}+\mathrm{PSS}^{-} \mathrm{M}^{+}
$$

PEDOT:PSS can be processed as a water dispersion using traditional printing and coating tools, which makes this electrochemical colorant an excellent candidate as the pixel electrode material in for instance paper-based displays [12]. By comparing conventional inorganic-based EC displays that have been developed during the last three decades $[13,14]$, one of the greatest advantages of using PEDOT:PSS in printed paper displays is that the material can serve as the electrical wires to route the updating signals, as both the counter and pixel electrodes in the actual display cells, and 
also as the conductivity switching material in electrochemical transistors. All these features are required to assemble an integrated matrix-addressed display device. Reducing the variety and number of materials directly leads to a reduction of manufacturing costs and simple device architectures; characteristics that are crucial to achieve low-cost and easily produced displays for large-area and/or single-use applications. In general, several performance specifications must be reached in order to successfully apply reflective printed displays in real products, such as the color switching time, power dissipation, long-term cycle/storage lifetime, color variations and color switch contrast. High color contrast is important in order to reach good perception of images and texts in a variety of ambiences, not only direct sunlight but also indoors. Much research efforts have specifically been devoted to improve the color contrast of EC polymers, primarily including efforts of tailor-making the polymer chemical structures that display large optical absorption differences upon electrochemical switching [15,16,17,18,19,20,21].

Here, we describe a route to optimize the color contrast in EC displays manufactured according to a device structure in which the active materials of both electrodes are made from PEDOT:PSS, see Figure 1 (a). In order to simulate and evaluate the color contrast of reflective displays, coloration efficiency or transmittance measurements are often employed [22,23], but here a colorimetric method based on the CIE $\mathrm{L}^{*} \mathrm{a}^{*} \mathrm{~b}^{*}$ color system is used [24,25]. In contrast to e.g. RGB and CMYK color spaces, the CIE L*a*b* color space approximates the human vision by its perceptual uniformity and is therefore often used as the color standard in many industrial applications. This color space has three parameters, $\mathrm{L}^{*}, \mathrm{a}^{*}$ and $\mathrm{b}^{*}$, that represent lightness, red-green chromaticity and yellow-blue chromaticity, respectively. $\mathrm{L}^{*}$ is normalized from 0 (dark black) to 100 (bright white), while $\mathrm{a}^{*}$ and $\mathrm{b}^{*}$ have no numeral limitations. Positive and negative $\mathrm{a}^{*}$ corresponds to red and green color, respectively, while positive and negative $b^{*}$ represents yellow and blue, respectively. The manufacturing process of the EC display studied here is simple; the 
display is constructed from a pair of planar PEDOT:PSS electrodes sandwiching a whitish, opaque and gelled electrolyte. One of the electrodes defines the pixel electrode, while the other serves as the hidden counter electrode located under the opaque electrolyte. The optical properties of the pixel electrode are measured at the same time as an addressing voltage is applied to both electrodes. When a negative potential is applied to the pixel electrode PEDOT:PSS appears dark blue (electrochemically reduced to its neutral state) to the human eye, while it becomes semi-transparent as it is positively biased (electrochemically oxidized), according to (1). Note that the semi-transparent oxidized state of the PEDOT:PSS layer will result in a white appearance of the reflective display cell. This is due to that the opaque pigment within the electrolyte scatters any incoming light. The topic in this paper aims at optimizing the color contrast by varying the thicknesses of the two electrodes. Varying the thicknesses impact the overall charge capacity of the electrochemical cell at the same time as it controls the optical absorption of the pixel electrode.

2. Materials and methods

2.1 Preparation of EC display devices

1.3 wt.-\% PEDOT:PSS coating dispersion (Baytron P HC), diethyleneglycol (DEG) [26] and fluorosurfactant (Zonyl FS-300) were purchased from H.C. Starck, Sigma-Aldrich and DuPont, respectively, and were used as received without further purification. The three material components were mixed according to a weight ratio of PEDOT:PSS/DEG/FS-300 =95/5/0.1 wt.- $\%$ and then extensively stirred (hereinafter called coating dispersion). Transparent polyethylene terephthalate (PET, Polifoil Bias) films were purchased from Policrom Screen and were used as the solid substrate carrying the display components. The coating dispersion was deposited onto the PET substrate using a wire bar, along with drying at $120{ }^{\circ} \mathrm{C}$ for $4-5$ minutes. In some display samples, the PEDOT:PSS coating and drying processes were repeated in order to achieve thicker layers. 
Different wire bars were used in this study to vary the thickness of the PEDOT:PSS layers. They were purchased from R.D. Specialties (US) and the wire bar numbers of 12, 26 and 50 were used;

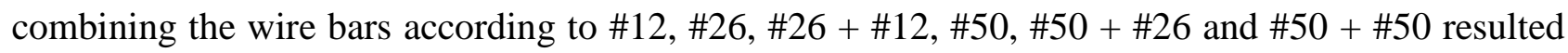
in the six different coating thicknesses $255,513,787,988,1381$ and $2081 \mathrm{~nm}$ respectively. It was found that the PEDOT:PSS thickness did not increase perfectly linear with respect to the number of coating steps for a specific wire bar. This is possibly due to that a slightly lower pressure was applied to the wire bar during the second coating step or that the surface tension of the underlying material varies between the first and second coating procedure, i.e. the PET and the PEDOT:PSS surfaces exhibit different wetting characteristics. The actual pixel electrode dimension was defined to $1 \times 1 \mathrm{~cm}^{2}$ using a plastic masking film. Before masking, a line of an electronically conducting carbon paste (7102 purchased from DuPont, dried at $120{ }^{\circ} \mathrm{C}$ for 2 minutes) was manually deposited from the edge of the PET substrate towards the opening of the plastic masking film on each pixel and counter electrode in order to minimize the potential drop along the coated PEDOT:PSS layer. Next, an electrolyte solution was prepared; sodium poly(styrene sulfonate) (PSSNa, M.W. 70k), D-sorbitol and glycerol were purchased from Alfa Aesar, Sigma-Aldrich and Merck, respectively, and were used as received. The compounds were mixed into the same aqueous solution according to the weight ratio of PSSNa/D-Sorbitol/glycerol/deionized water $=40 / 10 / 10 / 40$ wt.- $\%$. After thorough mixing and stirring, 5 wt.- $\%$ of $\mathrm{TiO}_{2}$ powder (KRONOS2300 purchased from KRONOS) was carefully mixed into the aqueous electrolyte system to serve as the white opaque pigment. The white electrolyte was deposited onto both the pixel electrode and the counter electrode, followed by drying for 1 minute at $60{ }^{\circ} \mathrm{C}$. The two stacks, wherein each stack was consisting of a foil, an electrode and an electrolyte, were then laminated into a vertical EC display device by utilizing the sticky white electrolyte gel as the glue, see Figure 1 (b). 
2.2 Characterization of the manually deposited PEDOT:PSS coatings

PEDOT:PSS layers of different thickness, coated by using the wire bars, were characterized using UV-visible-NIR spectroscopy (Perkin Elmer Lambda 900 Spectrometer) and an optical profilometer (Sensofar PLu neox, ePSI (extended phase shift interference) and $\times 20$ objective lens). The surface roughness, Ra, was evaluated by the Sensofar optical profilometer.

\subsection{Color contrast measurements}

The display devices were biased with a DC voltage that was applied from a Keithley SourceMeter model 2400 and the pixel electrode was defined as the electrical ground. This gives that by applying a positive voltage to the display device results in reduction of the pixel electrode, i.e. the display switches to a dark blue color. For each applied voltage bias, a colorimetric analysis of the EC display cell is performed. This includes measuring the values of $\mathrm{L}^{*}, \mathrm{a}^{*}$ and $\mathrm{b}^{*}$ of the pixel electrode. The colorimetric characterization was performed using a Spectrophotometer (Datacolor Mercury, aperture diameter of $6.5 \mathrm{~mm}$ for the illumination, aperture diameter of $2.5 \mathrm{~mm}$ for the measurement, SCE (specular component excluded) mode and D65/10 degrees illumination). Finally, the color contrast value $\Delta \mathrm{E}^{*}$ for each applied potential was calculated from:

$$
\Delta \mathrm{E}^{*}=\left[\left(\mathrm{L}_{2}{ }_{2}-\mathrm{L}_{1}\right)^{2}+\left(\mathrm{a} *_{2}-\mathrm{a} *_{1}\right)^{2}+\left(\mathrm{b}^{*}{ }_{2}-\mathrm{b} *_{1}\right)^{2}\right]^{1 / 2}
$$

Here $\mathrm{L}_{2}, \mathrm{a}_{2}$ and $\mathrm{b}_{2}{ }_{2}$ represent the color space parameters when the pixel electrode is switched on, i.e. the PEDOT:PSS of the pixel electrode is electrochemically reduced and switched to its blue state, and $\mathrm{L}^{*}{ }_{1}, \mathrm{a}^{*}{ }_{1}$ and $\mathrm{b}^{*}{ }_{1}$ represent the color space parameters when the pixel electrode is switched to its semi-transparent state, i.e. PEDOT:PSS is electrochemically oxidized.

\subsection{Switching time measurements}

EC displays having an area of $1.5 \times 1.5 \mathrm{~mm}^{2}$ were manufactured and were used for 
switching time measurement. One reference display was manufactured by using a commercial PEDOT:PSS coating on PET foil, Orgacon EL-350 from AGFA, as both the pixel and the counter electrode. The other display was consisting of bar coated PEDOT:PSS layers; a thickness of $\sim 500$ $\mathrm{nm}$ for the pixel electrode and a thickness of $\sim 2100 \mathrm{~nm}$ for the counter electrode. The color switching time measurement was performed by using a laser diode in conjunction with a photodiode, see Figure 1 (c). The display was irradiated by a laser diode peaking at $650 \mathrm{~nm}$, a wavelength that matches the absorption peak of the reduced state of PEDOT:PSS, and the scattered light was detected by the photodiode (Hamamatsu S1337-66, $5.8 \times 5.8 \mathrm{~mm}^{2}$ active area) and the photocurrent was recorded using a Keithley 2400 SourceMeter. The display was covered by a black film having a precut $1 \times 1 \mathrm{~mm}^{2}$ hole defining the measured display area, such that the remaining black film increased the signal-to-noise ratio.

(a)

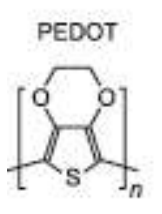

(b)

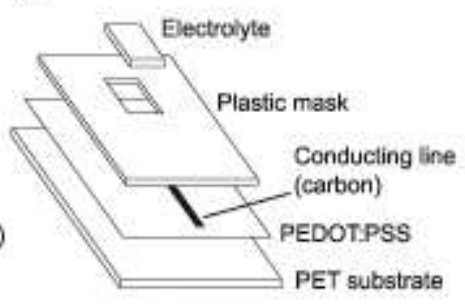

(c)

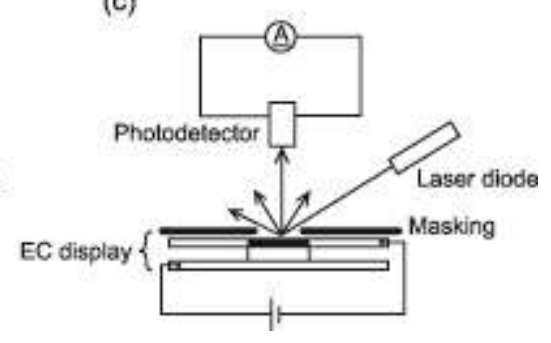

Figure 1. (a) Chemical structures of PEDOT and PSS (acid and sodium salt). (b) A drawing showing one of the EC display electrodes; two similar electrodes are then laminated to complete the EC display. (c) The experimental setup for the switching time measurement.

3. Results and discussion

3.1 Characterization of the manually deposited PEDOT:PSS coatings

From the optical absorption measurements a linear relationship between the polymer 
thickness and absorbance was observed. The resulting absorbance spectra and the absorbance vs. polymer thickness plots are shown in Figure 2. The optical absorption was recorded at a wavelength of $640 \mathrm{~nm}$ since this is the absorption peak for the reduced state of PEDOT:PSS. The thickness data were estimated by using the optical profilometer, see Figure 3 that shows the two thickest PEDOT:PSS coatings. A brief evaluation of the surface topography was also performed using the optical profilometer, with the result that $100 \times 100 \mu \mathrm{m}^{2}$ areas of manually coated PEDOT:PSS films and Orgacon EL-350 both showed about the same surface roughness; Ra 5-10 $\mathrm{nm}$. The inset graph of Figure 2 shows the actual optical absorption spectra of the coated films. The average thickness values were 255, 513, 787, 988, 1381 and $2081 \mathrm{~nm}$, based on measurements on five different locations on each coated film, and the corresponding absorbance values were 0.098, 0.206, $0.311,0.402,0.530$ and 0.836 , respectively.

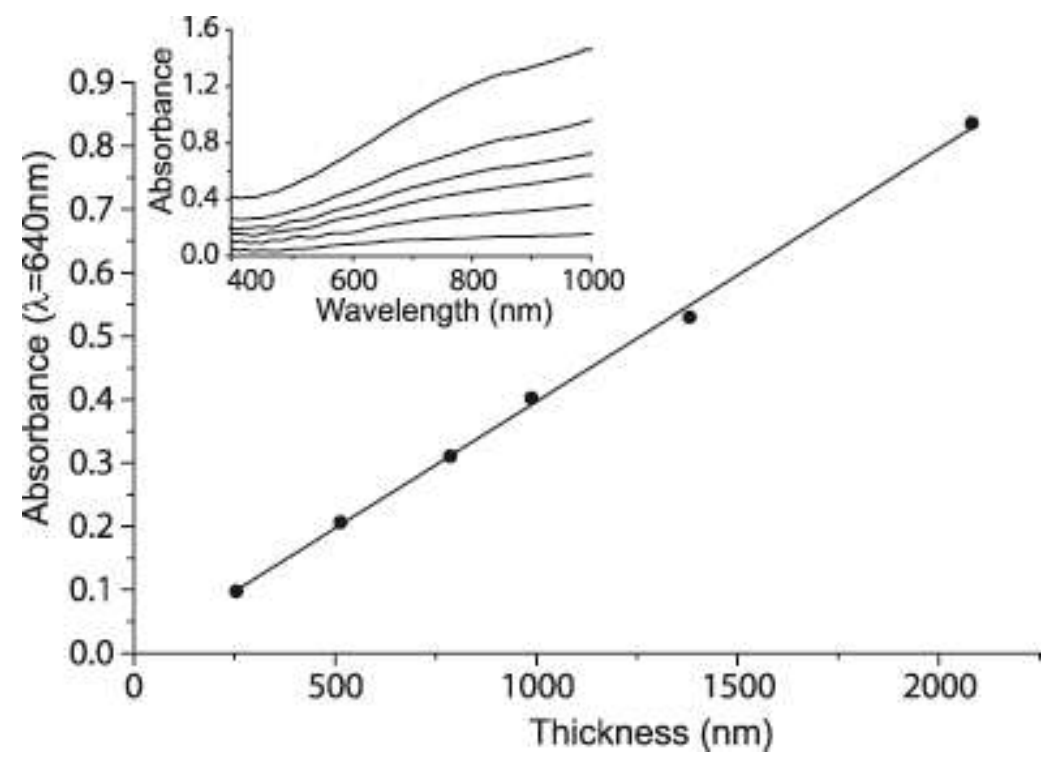

Figure 2. The absorbance vs. thickness dependence is illustrated, where the absorbance values were recorded at $640 \mathrm{~nm}$. The inset shows the actual optical absorption spectra of the PEDOT:PSS coatings. 


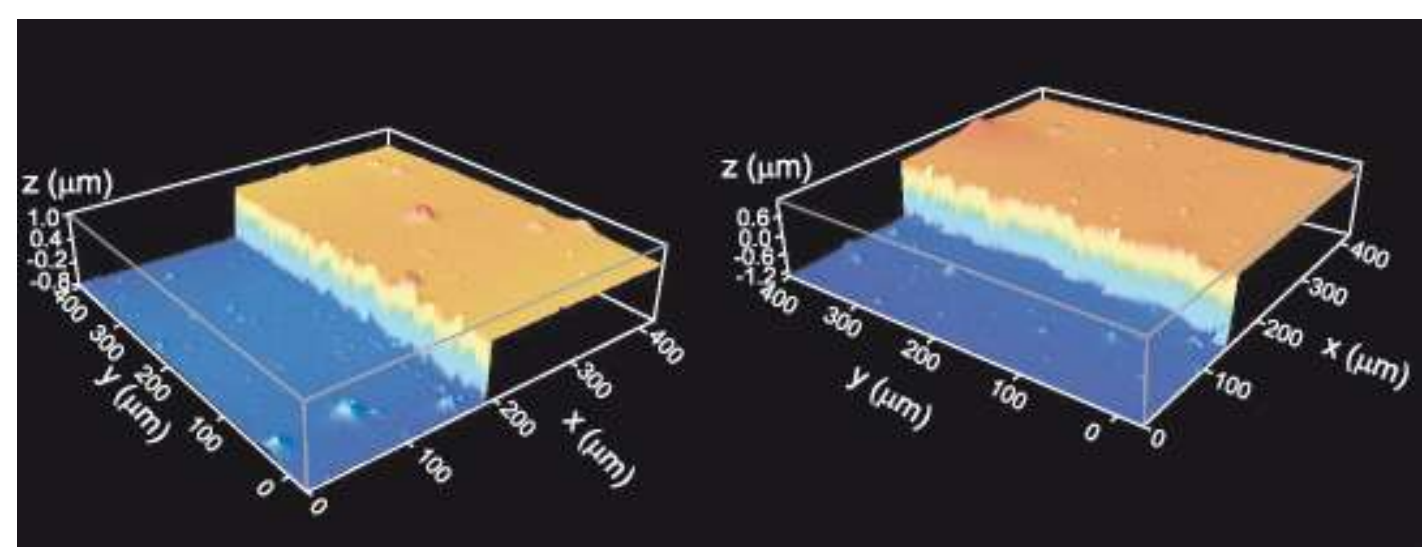

Figure 3. Images recorded by the optical profilometer provides the thicknesses of the PEDOT:PSS films.

3.2 Thickness dependence of the color contrast

\subsubsection{Optimizing the thickness of the pixel electrode}

The change in color switch contrast was characterized by varying the thickness of the pixel electrode. The counter electrodes reported in section 3.2.1 were all formed by depositing droplets of the PEDOT:PSS coating dispersion onto the plastic substrate. By doing this, we ensure that the resulting counter electrode is always sufficiently thick to enable full coloration for all variations of the evaluated pixel electrodes [27]. By varying the thickness of the pixel electrode, it was confirmed that thin active polymer layers serving as the pixel electrode became relatively more transparent in both the neutral and oxidized state, and vice versa. High color switch contrast was of course obtained if the oxidized and reduced state of the pixel electrode showed high transparency (the whitish opaque electrolyte dominates the reflection characteristics of the display) and strong absorption (dark blue), respectively. Hence, the highest color switch contrast could be obtained by optimizing the thickness of the pixel electrode. The measured $\mathrm{L}^{*}$ values and the calculated $\Delta \mathrm{E}^{*}$ for the colored and the white states of the displays were plotted as a function of the polymer thickness of the pixel electrode, see Figure 4. It was found that the display sample having a pixel electrode 
thickness of approximately $500 \mathrm{~nm}$ (manufactured using the \#26 wire bar) resulted in the highest color switch contrast. EC displays including a pixel electrode that is thinner than $500 \mathrm{~nm}$ could not be switched to the desired dark blue color, although their oxidized states appeared white due to that the pixel electrode exhibited very good transparency characteristics. Pixel electrodes with a thickness exceeding $500 \mathrm{~nm}$ were clearly exhibiting a relatively more deep blue color in the reduced state, but were instead not transparent enough in the oxidized state. Hence, the optimized pixel electrode thickness was found to be in the range of approximately 500-600 $\mathrm{nm}$ and the resulting color switch contrast reaches $\Delta \mathrm{E}^{*} \sim 42$, see Figure 4 .

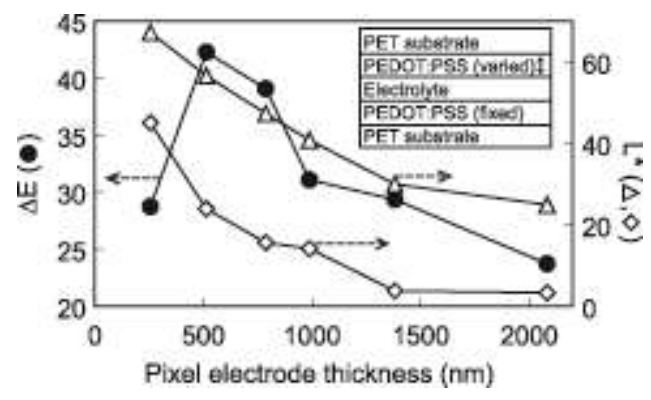

Figure 4. Color contrast $\left(\Delta \mathrm{E}^{*}\right)$ and lightness $\left(\mathrm{L}^{*}\right)$ vs. the PEDOT:PSS thickness of the pixel electrode. Black filled circles represent $\Delta \mathrm{E}^{*}$, while triangles and diamonds denote the $\mathrm{L}^{*}$ values of the white and colored state, respectively. A bias of $+2.2 \mathrm{~V}$ and $-1.2 \mathrm{~V}$ was used to obtain the electrochromic color switch between the fully reduced and the fully oxidized states. The inset shows the cross section image of the EC display used in this measurement.

\subsubsection{Optimizing the thickness ratio between the counter and pixel electrodes}

Next, the counter electrode thickness was optimized while keeping the thickness of the pixel electrode constant at approximately $500 \mathrm{~nm}$ (bar \#26). PEDOT:PSS is semi-oxidized in its pristine state. Here, we aimed at determining the necessary thickness of the counter electrode in order to obtain complete reduction, and hence also deepest possible blue color, of the pixel 
electrode. In this measurement the applied voltage was increased in steps of $200 \mathrm{mV}$, see Figure 5. As a result, the highest $\Delta \mathrm{E}^{*}$ color contrast value was achieved when the counter electrode was approximately four times thicker than the pixel electrode. When comparing this optimized counter electrode with an EC display device having Orgacon EL-350 as both the pixel and counter electrode (data not shown), the $\Delta \mathrm{E}^{*}$ value was improved by $47 \%$, i.e. from 28.66 to 42.26 .
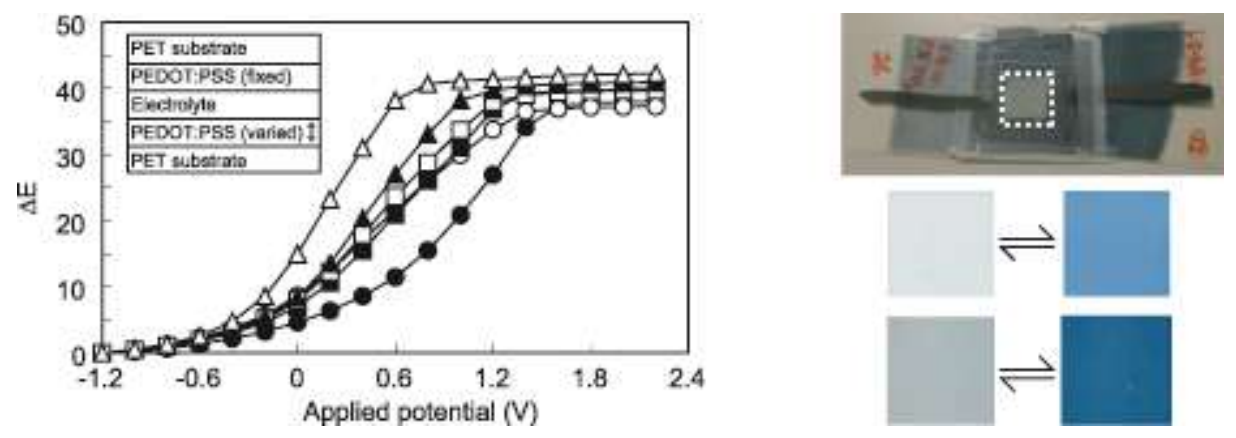

Figure 5. Left: Graphs showing the color contrast of various counter electrode thicknesses as a function of the applied potential. The pixel electrode thickness is here kept constant and the thickness ratios between the pixel and counter electrodes are $1: 0.5$ for the data represented by filled circles, $1: 1$ (open circles), $1: 1.5$ (filled squares), $1: 2$ (open squares), $1: 3$ (filled triangles) and $1: 4$ (open triangles). The inset shows the cross section image of the EC display used in this measurement. Right: The photograph shows the entire display device (top) along with images scanned by a flatbed scanner (middle and bottom). The scanned images correspond to $0.8 \times 0.8 \mathrm{~cm}^{2}$ of the $1 \times 1 \mathrm{~cm}^{2}$ total display area (white dashed line). The two lower images originate from the display device having the highest contrast, while the two images in the middle are based on a display device consisting of Orgacon EL-350 electrodes. The photos to the left are EC pixel electrodes switched to the oxidized state of the displays, while the two photos to the right are from EC displays switched to their reduced state.

Additionally, from the data of Figure 5 it is observed that displays having a relatively thick 
counter electrode show an increased color switch contrast sensitivity vs. the applied potential, i.e. the $\Delta \mathrm{E}^{*}$ color switch contrast value saturates already for a voltage $<\sim 0.8 \mathrm{~V}$, while display samples having a relatively thin counter electrode typically require higher voltages to reach the same color contrast value. For example, for an applied potential equal to $0.6 \mathrm{~V}$, the color switch contrast $\Delta \mathrm{E}^{*}$ values increased by $170 \%$ and $82 \%$ when the counter electrode had a thickness of approximately $2100 \mathrm{~nm}$ as compared to a counter electrode thickness of 250 and $500 \mathrm{~nm}$, respectively. This means that both the required voltage and the power consumption can be lowered by utilizing a relatively thick counter electrode in the EC displays. Conversely, when a relatively thin counter electrode is used, the color switching behavior of the EC display occurs more moderately vs. increased voltage. An applied voltage exceeding $\sim 1.4 \mathrm{~V}$ is required in order to reach color saturation when using the thinnest counter electrode, see Figure 5.

\subsection{Measuring the color switch time of the EC display}

The optimized EC display cell, having a pixel electrode thickness of $500 \mathrm{~nm}$ and a 2100 nm thick counter electrode, was further evaluated with respect to the color switch time characteristics. The result was then compared with a reference EC display in which Orgacon EL-350 was used as both electrodes. The switching time measurements, which are shown in Figure 6, resulted in an estimated switching time of $53 \mathrm{~ms}$ for the reference EC display and $41 \mathrm{~ms}$ for the optimized EC display. This gives that for the same driving conditions, the color switch dynamics of the optimized EC display cell is similar, or actually slightly faster, as compared to the reference EC display. One plausible disadvantage of having a very thick display counter electrode, i.e. having one half-cell with a large ion storage capacity, is that it could impact the switch time properties negatively, however, this was obviously not observed. 

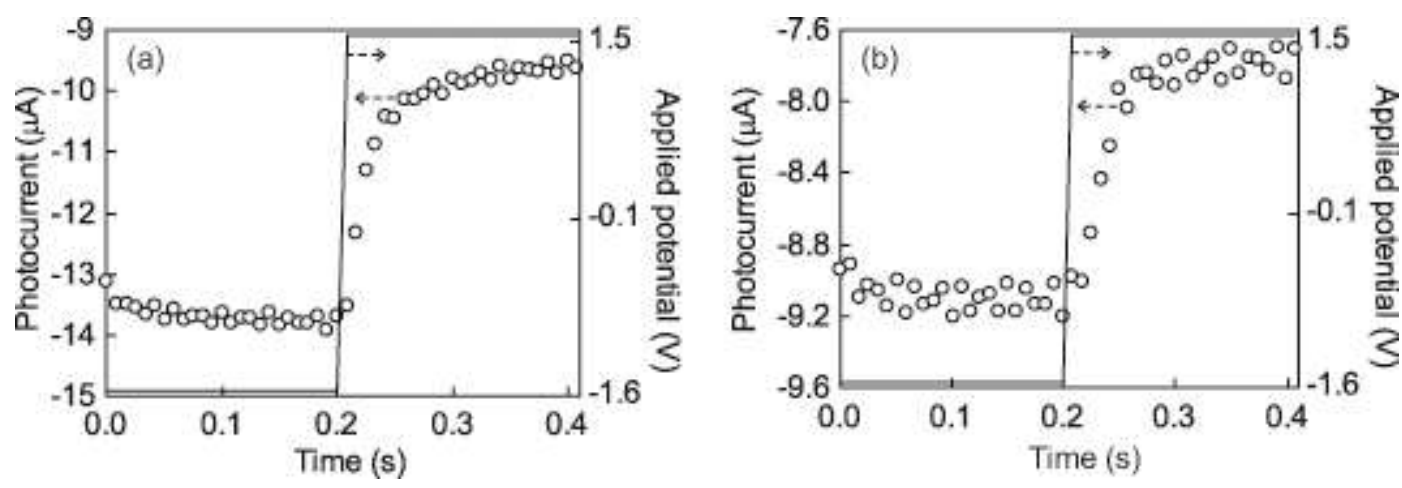

Figure 6. The switching times of the EC displays consisting of (a) Orgacon EL-350 on both electrodes and (b) bar coated PEDOT:PSS electrodes for optimized color switch contrast. The time delay between the data points is $8 \mathrm{~ms}$ and the applied voltage (solid lines) was scanned from -1.5 $\mathrm{V}$ (white color) to $1.5 \mathrm{~V}$ (blue color). The recorded output current from the photodiode corresponds to the absorbance switching of the EC displays. The reported switching times correspond to $80 \%$ of the difference in photocurrent between the white and blue-colored states.

\section{Conclusion}

We report on a strategy to optimize the color switch contrast of organic electrochromic displays by carefully balancing the thickness of the pixel electrode and counter electrode, independently. By utilizing a PEDOT:PSS thickness of $\sim 500$ and $\sim 2000 \mathrm{~nm}$ on the pixel and the counter electrode, respectively, results in an optimized color switch contrast of $\Delta \mathrm{E}^{*} \sim 42$, which corresponds to an increase of $\sim 50 \%$ as compared to previous results where both electrodes were made from standard coatings of the same thickness. Additionally, we also demonstrated that the EC display showed similar, or actually slightly shorter, switch time characteristics, as compared to the reference display cells. The findings add a simple tool to the box of strategies in order to improve the color switch contrast of reflective electrochromic displays. Prior strategies have primarily included optimization of the chemical structure and the design of new display architectures. 


\section{Acknowledgement}

The authors gratefully acknowledge the Centerprise project at VINNOVA (research and innovation for sustainable growth in Sweden) for funding of this research.

1 C. K. Chiang, C. R. Fincher, Jr., Y. W. Park, A. J. Heeger, H. Shirakawa, E. J. Louis, S. C. Gau, A. G. MacDiarmid, Phys. Rev. Lett. 39 (1977) 1098-1101.

2 J. Roncali, Chem. Rev. 97 (1997) 173-206.

3 R. D. McCullough, S. Tristram-Nagle, S. P. Williams, R. D. Lowe, M. Jayaraman, J. Am. Chem. Soc. 115 (1993) 4910-4911.

4 PEDOT:PSS screen printing paste "CLEVIOS PH-1000" manufactured by Heraeus, $<$ http://clevios.com/en/downloads/heraeus-conductive-polymers-downloads.aspx >

5 F. Louwet, L. Groenendaal, J. Dhaen, J. Manca, J. Van Luppen, E. Verdonck, L. Leenders, Synth. Met. 135-136 (2003) 115-117.

6 G. Heywang, F. Jonas, Adv. Mater. 4 (1992) 116-118.

7 L. Groenendaal, F. Jonas, D. Freitag, H. Pielartzik, J. R. Reynolds, Adv. Mater. 12 (2000) 481-494.

8 S. Kirchmeyer, A. Elschner, K. Reuter, W. Lovenich, U. Merker, PEDOT: principles and applications of an intrinsically conductive polymer, first ed., CRC Press, Boca Raton, 2010.

9 S. Y. Park, J. M. Lee, N. Noh, S. U. Son, J. Mater. Chem. (2009) 19 7959-7964.

10 R. J. Mortimer, Annu. Rev. Mater. Res. (2011) 41 241-268.

11 A. A. Argun, M. Berard, P.-H. Aubert, J. R. Reynolds, Adv. Mater. (2005) 17 422-426

12 P. Andersson, D. Nilsson, P.-O. Svensson, M. Chen, T. Remonen, T. Kugler, M. Berggren, Adv. Mater. 14 (2002) $1460-1464$.

13 J. V. Gabrusenoks, P. D. Cikmach, A. R. Lusis, J. J. Kleperis, G. M. Ramans, Solid State Ionics 14, (1984) 25-30.

14 D. S. Chung, J. W. Kim, Y. J. Yi, M. H. Jung, C. H. Noh, S. J. Jeon, R. R. Das, B. G. Song, Y. W. Jin, S. Y. Lee, 2010 23rd Annu. Meet. IEEE Photonics Soc., PHOTONICS 2010, art. no. 5698880, pp. 303-304.

15 P. M. Beaujuge, S. Ellinger, J. R. Reynolds, Nat. Mater. 7 (2008) 795-799.

16 P. Shi, C. M. Amb, E.P. Knott, E. J. Thompson, D. Y. Liu, J. Mei, A. L. Dyer, J. R. Reynolds, Adv. Mater. 22 
(2010) 4949-4953.

17 S. Hellström, P. Henriksson, R. Kroon, E. Wang, M. R. Andersson, Org. Electron. 12 (2011) 1406-1413.

18 D. Corr, U. Bash, D. Fay, M. Kinsella, C. McAtamney, F. O’Reilly, S. N. Rao, N. Stobie, Solid State Ionics 165 (2003), 315-321.

19 A. L. Dyer, M. R. Craig, J. E. Babiarz, K. Kiyak, J. R. Reynolds, Macromolecules (2010), 43, 4460-4467.

20 J. Padilla, Sol. Energy Mater. Sol. Cells (2011), doi:10.1016/j.solmat.2011.06.019.

(Article in press as of Sept 12)

21 C. L. Gaupp, D. M. Welsh, J. R. Reynolds, Macromol. Rapid Commun. (2002) 23 885-889.

22 J. Padilla, V. Seshadri, G. A. Sotzing, T. F. Otero, Electrochem. Commun (2007) 9 1931-1935.

23 J. Padilla, T. F. Otero, Electrochem. Commun. (2008) 10 1-6.

24 B. C. Thompson, P. Schottland, K. Zong, and J. R. Reynolds, Chem. Mater. 12 (2000) 1563-1571.

25 R. J. Mortimer, K. R. Graham, C. R. G. Grenier, J. R. Reynolds, ACS Appl. Mater. Interfaces, (2009), 1, 10, $2269-2276$

26 X. Crispin, F. L. E. Jakobsson, A. Crispin, P. C. M. Grim, P. Andersson, A. Volodin, C. van Haesendonck, M. Van der Auweraer, W. R. Salaneck, M. Berggren, Chem. Mater. 18 (2006) 4354-4360.

27 P. Andersson, R. Forchheimer, P. Tehrani, M. Berggren, Adv. Funct. Mater. 17 (2007) 3074-3082. 